\title{
Caractéristiques physicochimiques de quelques matières premières utilisées dans la formulation des aliments pour volaille au Burkina Faso
}

Inoussa KY1*, Charles PARKOUDA², Marius K. SOMDA¹ ; Bréhima DIAWARA² ; Mamoudou H. DICKO ${ }^{1}$ 1 Université Joseph Ki-Zerbo, UFR/SVT, Laboratoire de Biochimie, Biotechnologie, Technologie Alimentaire et Nutrition (LABIOTAN) 03 BP 7021 Ouagadougou 03, Burkina Faso

2 Centre National de la Recherche Scientifique et Technologique (CNRST), Institut de Recherche en Sciences Appliquées et Technologies (IRSAT), Département Technologie Alimentaire (DTA).

*Correspondance : E-mail: inoussaky2005@yahoo.fr Tel: +22678653591 /+22671845984

Original submitted in on $02^{\text {th }}$ June 2020. Published online at www.m.elewa.org/journals/ on $31^{\text {st }}$ July 2020 https://doi.org/10.35759/JABs.151.8

\section{RESUME}

Objectifs: Connaitre la composition physicochimique des matières premières les plus utilisées dans la formulation des aliments pour volaille.

Méthodologie et Résultats : Une étude prospective a permis de répertorier les matières alimentaires les plus utilisées pour l'alimentation de volaille au Burkina Faso. Les principaux constituants ont été déterminés par les méthodes AOAC. Les acides aminés et les minéraux ont été déterminés respectivement par la chromatographie et la spectrométrie à flamme. Les résultats ont montré des proportions massiques $(\mathrm{g} / 100 \mathrm{~g}$ MS) comprises entre $9,17 \pm 0,13$ et $59,9 \pm 0,27$ pour les protéines, 2,24 $\pm 0,10$ et 15,56 $\pm 0,03$ pour les lipides, $4,16 \pm 0,04$ et $73,8 \pm 0,07$ pour les carbohydrates totaux, entre $6,04 \pm 0,13$ et $14,53 \pm 0,07$ pour la cellulose, $0,01 \pm 0,00$ et $34,03 \pm 0,07$ pour le calcium, $0,02 \pm 0,07$ et $18,01 \pm 0,00$ pour le phosphore. Les teneurs en méthionine, lysine, thréonine étaient respectivement comprises entre $0,19 \pm 0,02$ et $1,49 \pm 0,03 ; 0,25 \pm 0,06$ et $2,38 \pm 0,03$, et $0,30 \pm 0,03$ et 2,32 $\pm 0,03$.

Conclusion et application des résultats: II ressort de cette étude que la farine de poisson présente les teneurs les plus élevées en protéine $(59,9 \pm 0,27 \mathrm{~g} / 100 \mathrm{~g})$, calcium $(6,42 \pm 0,04 \mathrm{~g} / 100 \mathrm{~g})$ et phosphore $(3,88 \pm 0,06$ $\mathrm{g} / 100 \mathrm{~g})$. Le soja est le plus riche en matière grasse $(17,98 \pm 0,10 \mathrm{~g} / 100 \mathrm{~g})$ et le maïs jaune est le plus riche en glucide $(73,8 \pm 0,07 \mathrm{~g} / 100 \mathrm{~g})$. Ces données produites peuvent servir à l'établissement de tables de composition des matières premières locales utilisables dans l'alimentation de volaille et leur vulgarisation auprès des aviculteurs leur permettra de mieux s'orienter dans le choix des ingrédients de hautes valeurs nutritionnelles et de réduire le coût de la formulation.

Mots clés : Formulation d'aliment, volaille, composition, nutrition

\begin{abstract}
Objectives: To know the physicochemical composition of the raw materials mostly used in the formulation of poultry feed.

Methodology and Results: A prospective study was carried out to identify the most commonly used feed materials for poultry feed in Burkina Faso. The main constituents were determined by AOAC methods. Amino acids and minerals were determined respectively by chromatography and flame spectrometry. Results
\end{abstract}


showed mass proportions ( $\mathrm{g} / 100 \mathrm{~g} \mathrm{DM}$ ) between $9.17 \pm 0.13$ and $59.9 \pm 0.27$ for proteins, $2.24 \pm 0.10$ and $15.56 \pm 0.03$ for lipids, $4.16 \pm 0.04$ and $73.8 \pm 0.07$ for total carbohydrates, $6.04 \pm 0.13$ and $14.53 \pm 0.07$ for cellulose, $0.01 \pm 0.00$ and $34.03 \pm 0.07$ for calcium, $0.02 \pm 0.07$ and $18.01 \pm 0.00$ for phosphorus. Content in essential amino acids such as methionine, lysine, and threonine ranged between $0.19 \pm 0.02$ and $1.49 \pm$ $0.03,0.25 \pm 0.06$ and $2.38 \pm 0.03$, and $0.30 \pm 0.03$ and $2.32 \pm 0.03$, respectively.

Conclusion and application of results: It emerges from this study that fishmeal has the highest contents of protein $(59.9 \pm 0.27 \mathrm{~g} / 100 \mathrm{~g})$, calcium $(6.42 \pm 0.04 \mathrm{~g} / 100 \mathrm{~g})$ and phosphorus $(3.88 \pm 0.06 \mathrm{~g} / 100 \mathrm{~g})$. Soy is the richest in fat $(17.98 \pm 0.10 \mathrm{~g} / 100 \mathrm{~g})$ and yellow corn is the richest in carbohydrate $(73.8 \pm 0.07 \mathrm{~g} / 100 \mathrm{~g})$. These data produced can be used to establish composition tables for local raw materials that can be used in poultry feed and their popularization with poultry farmers will allow them to better orient themselves in the choice of ingredients with high nutritional values and reduce the cost of formulation

Keywords: Feed Formulation, poultry, composition, nutrition

\section{INTRODUCTION}

Au Burkina Faso, l'élevage occupe une grande partie de la population du pays et représente environ 10 à $20 \%$ du PIB du pays et est le deuxième plus grand contributeur à la valeur ajoutée agricole, après le coton (FAO, 2018). L'élevage avicole est très répandu au Burkina Faso et fournit les moyens de subsistance à environ 1,6 million de producteurs et de ménages, en particulier les femmes des zones rurales (Ayssiwede et al., 2013; FAO, 2018). Avec une contribution d'environ $6 \%$ du PIB agricole, l'aviculture joue un rôle majeur dans l'économie du Burkina Faso (FAO, 2018). En aviculture, l'alimentation est le principal facteur qui affecte le plus la rentabilité économique d'exploitation car elle peut représenter $70 \%$ des charges variables (Oladokun \& Johnson, 2012), . La maîtrise des matières premières utilisées et des méthodes de formulation sont nécessaires pour maintenir un niveau de profit satisfaisant (Afolayan \& Afolayan, 2008). La fabrique d'aliment consiste à mélanger plusieurs ingrédients disponibles en tenant compte de leur taux d'incorporation (Almasad et al., 2011). L'aliment doit apporter des nutriments necessaire pour couvrir les besoins nutritionnels de la volaille en adéquation avec des objectifs de production (Saxena, 2012). La réussite d'une formulation d'aliment de qualité dépend fortement d'une bonne connaissance des caractéristique physicochimiques des matières premières (Ponka et al., 2017). Contrairement aux pays occidentaux qui disposent des tables publiques de composition des matières premières alimentées par une masse considérable de résultats d'analyses (Ngom, 2004), le Burkina Faso ne dispose à l'heure actuelle que très peu de données similaires. La formulation de rations alimentaires pour les volailles reste encore l'apanage des aviculteurs modernes. La caractérisation nutritionnelle des ingrédients, l'évaluation de l'apport nutritionnel nécessaire pour l'animal et la stratégie de formulation des aliments sont les étapes qui doivent être utilisés pour atteindre les objectifs (Pomar et al., 2009). Il y a des produits et sous-produits agricoles ou agroindustriels qui sont disponibles mais leur utilisation dans l'alimentation de la volaille constitue un handicap pour la plupart des aviculteurs locaux dû à la méconnaissance de la valeur nutritive des matières premières. Ainsi, dans l'optique de proposer à ces producteurs les ingrédients de bonne qualité au bas coût, le présent travail a pour objectif d'établir une base de données des valeurs nutritionnelles de quelques ingrédients entrant dans la formulation des aliments

\section{MATERIELS ET METHODES}


Matériel biologique: Le matériel biologique est constitué des échantillons de grains de maïs jaune, grains de maïs blanc, tourteau de coton, tourteau de soja, son de blé, son de riz, farine de poisson, coquille d'huitre et phosphate bicalcique. Ces matières premières ont été identifiées et sélectionnées sur la base de leur disponibilité sur le marché local, leur coût et leur seuil d'incorporation dans les aliments décrit par la bibliographie.

Echantillonnage : La collecte des échantillons a été faite auprès des fournisseurs livrant les ingrédients aux fermiers et au niveau d'une unité de transformation des aliments (Société de Fabrique d'aliments pour Bétail SOFAB -sa). Les échantillons ont été conditionnés dans des emballages plastiques en polyéthylène de couleur sombre pour empêcher l'absorption d'eau et l'oxydation des lipides en présence de la lumière. Par la suite, ils ont été transportés au Laboratoire pour les analyses chimiques.

Méthodes de détermination de la composition biochimiques : La teneur en protéine des échantillons a été déterminée au Kjeldahl selon la méthode AOAC 979.09 (AOAC, 1999), qui consiste à minéraliser l'azote protéique en ammoniac pour le doser ensuite par acidimétrie. La détermination des matières grasses a été faite par extraction au Soxhlet selon la méthode AOAC. 960.39 (AOAC, 1999) en utilisant l'hexane comme solvant. Les glucides ont été dosés par la méthode spectrophotométrique de Montreuil et Spik (1969). Ainsi, 0,2 g de broyat de chaque matière première a été additionné de $2 \mathrm{ml}$ de solution d'orcinol et

\section{RESULTATS}

La composition en macronutriments et micronutriments varie en fonction des matières premières (Tableaux 1 et 2). Les teneurs en protéine varient de $9,07 \pm 0,08$ (maïs blanc) à $59,9 \pm 0,27 \%(\mathrm{~m} / \mathrm{m})$ (farine de poisson). La farine de poisson, le tourteau de soja et le tourteau de coton sont les matières premières les plus riches en protéine avec des teneurs respectives de 59,9 $\pm 0,27$, $44,46 \pm 0,19$ et $39,84 \pm 0,22 \%(\mathrm{~m} / \mathrm{m})$. Les teneurs en matières grasses varient de $2,24 \pm 0,10$ à 17,98 $\pm 0,10$ $\%(\mathrm{~m} / \mathrm{m})$. Le soja et le son de riz sont les matières premières les plus riches en matière grasse avec des teneurs respectives de $17,98 \pm 0,10$ et 15,56 $\pm 0,03 \%$ $(\mathrm{m} / \mathrm{m})$. Les teneurs en glucide varient de 4,16 $\pm 0,04$ à $73,8 \pm 0,07 \%(\mathrm{~m} / \mathrm{m})$. Les maïs jaune et blanc sont les plus riches en amidon avec des teneurs respectives de $73,8 \pm 0,07$ et $66,57 \pm 0,45 \%(\mathrm{~m} / \mathrm{m})$. Les teneurs en cendres varient de 1,21 $\pm 0,14$ à 24,15 $\pm 0,57$. La farine de poisson est la matière première qui contient plus de
$7 \mathrm{ml}$ de solution de $\mathrm{H}_{2} \mathrm{SO}_{4}$ à $60 \%$, puis le mélange a été incubé au bain marie bouillant pendant $20 \mathrm{~min}$; les solutions sont placées à l'obscurité pendant 45 min puis à la température ambiante pendant $10 \mathrm{~min}$; la densité optique a été lue à $510 \mathrm{~nm}$. Les concentrations en glucide ont été déterminées à l'aide d'une courbe étalon de D-glucose.

La teneur en cendres a été déterminée selon la méthode AOAC 923.03 (AOAC, 1999) qui consiste à incinérer $5 \mathrm{~g}$ de l'échantillon au four à $550^{\circ} \mathrm{C}$, pendant 4 heures.

La teneur en cellulose brute a été déterminée par la méthode décrite par Aubry (2012). Le résidu est filtré dans un creuset en verre fritté, rincé abondamment, séché, pesé, calciné à $500^{\circ} \mathrm{C}$ puis repesé. La différence de poids correspond à la cellulose brute présente dans l'échantillon d'essai..

La teneur des minéraux (calcium et phosphore) des matières premières a été déterminée après minéralisation de l'échantillon selon la méthode décrite par Houba et al (1980).

Le profil et la quantité des acides aminés totaux a été déterminé par HPLC en phase inverse, en utilisant le système Pico-Tag décrit par Bindlingmeyer et al (1984). La valeur énergétique théorique a été calculée en utilisant les coefficients d'Atwater.

Analyses statistiques : Les résultats des analyses sont présentés sous forme de moyenne \pm écart-type. Le traitement des résultats s'est fait par analyse de variance (ANOVA) au seuil de significativité de $5 \%$. Ces analyses ont été effectuées avec le logiciel "XLSTAT version 7.5.2".

matières minérales. Les teneurs en cellulose varient de $6,04 \pm 0,13$ à $14,53 \pm 0,07 \%(\mathrm{~m} / \mathrm{m})$. Le tourteau de coton et le son de blé contiennent les plus fortes proportions de cellulose respectivement de $14,53 \pm 0,07$ et $14,34 \pm$ $0,06 \%(\mathrm{~m} / \mathrm{m})$. Les teneurs en calcium varient de $0,01 \pm$ 0,00 à $34,03 \pm 0,07 \%(\mathrm{~m} / \mathrm{m})$. Ce sont la coquille d'huitre et le phosphate bicalcique qui ont une forte concentration en calcium avec des teneurs respectives de $34,03 \pm 0,07$ et $25,54 \pm 0,05 \%(\mathrm{~m} / \mathrm{m})$. Les teneurs en phosphore varient de 0,02 $\pm 0,07$ à 18,01 $\pm 0,00 \%$ $(\mathrm{m} / \mathrm{m})$ et le phosphate bicalcique est la matière première la plus riche avec une teneur de 18,01 $\pm 0,00 \%(\mathrm{~m} / \mathrm{m})$. Enfin, les teneurs en acides, en méthionine, en lysine et en thréonine varient respectivement de 0,19 $\pm 0,02$ à $1,49 \pm 0,03 \%(\mathrm{~m} / \mathrm{m})$, de $0,25 \pm 0,06$ à 2,38 $\pm 0,03 \%$ $(\mathrm{m} / \mathrm{m})$ et $0,3 \pm 0,03$ à $2,32 \pm 0,03 \%(\mathrm{~m} / \mathrm{m})$. La farine de poisson, le tourteau de soja et le tourteau de coton sont les matières premières les plus riches en ces acides 
aminés essentiels. Les valeurs énergétiques des

échantillons varient de 194,5 à 400,6 kcal/100g.

Tableau 1 : Composition biochimique et valeur énergétique $(\mathrm{g} / 100, \mathrm{~m} / \mathrm{m}, \%)$

\begin{tabular}{l|l|l|l|l|l}
\hline Matières Premières & Protéines & Lipides & Glucides & Cendres & $\begin{array}{l}\text { Valeur énergétique } \\
\text { (kcal/100g) }\end{array}$ \\
\hline Mais Jaune & $9,17 \pm 0,13^{\mathrm{g}}$ & $4,28 \pm 0,15^{\mathrm{e}}$ & $73,8 \pm 0,07^{\mathrm{a}}$ & $1,26 \pm 0,12^{\mathrm{g}}$ & 370,4 \\
Mais Blanc & $9,07 \pm 0,08^{\mathrm{g}}$ & $4,04 \pm 0,12^{\mathrm{f}}$ & $66,57 \pm 0,45^{\mathrm{b}}$ & $1,21 \pm 0,14^{\mathrm{g}}$ & 339 \\
Soja & $33,5 \pm 0,25^{\mathrm{d}}$ & $17,98 \pm 0,10^{\mathrm{a}}$ & $33,84 \pm 0,18^{\mathrm{d}}$ & $4,21 \pm 0,31^{\mathrm{f}}$ & 431,1 \\
Tourteau de soja & $44,46 \pm 0,19^{\mathrm{b}}$ & $2,24 \pm 0,10^{\mathrm{g}}$ & $5,28 \pm 0,11^{\mathrm{f}}$ & $6,36 \pm 0,20^{\mathrm{d}}$ & 219,2 \\
Tourteau de coton & $39,84 \pm 0,22^{\mathrm{c}}$ & $3,93 \pm 0,1^{\mathrm{f}}$ & $4,16 \pm 0,04^{\mathrm{g}}$ & $7,08 \pm 0,20^{\mathrm{c}}$ & 211,4 \\
Farine de poisson & $59,9 \pm 0,27^{\mathrm{a}}$ & $9 \pm 0,17^{\mathrm{c}}$ & $\mathrm{ND}$ & $24,15 \pm 0,57^{\mathrm{a}}$ & 320,6 \\
Son de blé & $14,97 \pm 0,16^{\mathrm{e}}$ & $4,65 \pm 0,17^{\mathrm{d}}$ & $23,18 \pm 0,08^{\mathrm{e}}$ & $5,48 \pm 0,13^{\mathrm{e}}$ & 194,5 \\
Son de riz & $13,25 \pm 0,30^{\mathrm{f}}$ & $15,56 \pm 0,03^{\mathrm{b}}$ & $51,88 \pm 0,13^{\mathrm{c}}$ & $9,07 \pm 0,45^{\mathrm{b}}$ & 400,6 \\
\hline
\end{tabular}

Les valeurs reportées dans la même colonne et portant les lettres différentes en exposant sont significativement différentes au seuil de $5 \%$.

Tableau 2 : *Teneur en Cellulose, Calcium, Phosphore et en quelques acides aminés $(\%, \mathrm{~m} / \mathrm{m})$

\begin{tabular}{|c|c|c|c|c|c|c|}
\hline $\begin{array}{l}\text { Matières } \\
\text { Premières }\end{array}$ & Cellulose & Calcium & Phosphore & Méthionine & Lysine & Thréonine \\
\hline Mais Jaune & $9,66 \pm 0,04^{c}$ & $0,01 \pm 0,00 \mathrm{~g}$ & $0,3 \pm 0,03^{e}$ & $0,19 \pm 0,02^{e}$ & $0,25 \pm 0,06 \mathrm{e}$ & $0,3 \pm 0,039$ \\
\hline Mais Blanc & $9,69 \pm 0,05^{c}$ & $0,01 \pm 0,00 \mathrm{~g}$ & $0,28 \pm 0,03 e$ & $0,19 \pm 0,04 \mathrm{e}$ & $0,26 \pm 0,06 \mathrm{e}$ & $0,33 \pm 0,02$ \\
\hline Soja & $9,37 \pm 0,06^{d}$ & $0,18 \pm 0,00^{e}$ & $0,06 \pm 0,01^{f}$ & $0,56 \pm 0,05^{c}$ & $2,34 \pm 0,10^{a}$ & $1,67 \pm 0,04$ \\
\hline Tourteau de soja & $6,04 \pm 0,13^{f}$ & $0,3 \pm 0,03^{d}$ & $0,57 \pm 0,02^{d}$ & $0,61 \pm 0,03^{b}$ & $2,2 \pm 0,02^{b}$ & $1,77 \pm 0,11$ \\
\hline Tourteau de coton & $14,53 \pm 0,07^{a}$ & $0,15 \pm 0,00^{e}$ & $0,52 \pm 0,02^{d}$ & $0,64 \pm 0,03^{b}$ & $1,43 \pm 0,04^{c}$ & $2,22 \pm 0,04$ \\
\hline Farine de poisson & & $6,42 \pm 0,04^{c}$ & $3,88 \pm 0,06^{b}$ & $1,49 \pm 0,03^{a}$ & $2,38 \pm 0,03^{a}$ & $2,32 \pm 0,03$ \\
\hline Son de blé & $14,34 \pm 0,06^{b}$ & $0,07 \pm 0,00^{f}$ & $0,02 \pm 0,07^{f}$ & $0,28 \pm 0,03^{d}$ & $0,63 \pm 0,03^{d}$ & $0,56 \pm 0,04$ \\
\hline Son de riz & $7,96 \pm 0,11 \mathrm{e}$ & $0,07 \pm 0,00^{f}$ & $1,6 \pm 0,00^{c}$ & $0,29 \pm 0,03^{d}$ & $0,62 \pm 0,04^{d}$ & $0,43 \pm 0,04$ \\
\hline Coquille d'huitre & & $34,03 \pm 0,07^{a}$ & $0,07 \pm 0,03^{f}$ & ND & ND & ND \\
\hline $\begin{array}{l}\text { Phosphate } \\
\text { bicalcique }\end{array}$ & ND & $25,54 \pm 0,05^{b}$ & $18,01 \pm 0,00^{a}$ & ND & ND & ND \\
\hline
\end{tabular}

*Les moyennes situées dans la même colonne et portant les lettres différentes en exposant sont significativement différentes au seuil $P<0,05$. ND, non détecté.

\section{DISCUSSION}

La teneur en protéines du tourteau de soja est supérieure à la valeur $39,38 \pm 0,33 \mathrm{~g} / 100$ trouvée par Ponka et al. (2017) dans les tourteaux de soja au Cameroun. La teneur en protéines du soja est supérieure à celles qui sont disponibles dans la table de composition des aliments de la FAO (2019). Ces fortes teneurs en protéines peuvent être attribuées à la variété de soja utilisée. La teneur en protéines du tourteau de coton est relativement élevée par rapport aux données habituelles (Mongodin \& Rivière, 1965). Cela peut aussi être expliqué par la variété des grains de coton utilisés. Toutefois, la teneur en protéines de la farine de poisson est proche de la valeur $60 \%(\mathrm{~m} / \mathrm{m})$ trouvée dans la farine de poisson du Sénégal (Huart, 2004) mais reste largement inférieure à la valeur $76 \%$ trouvée dans les farines de poisson de France (Bastianeli et al., 2009).
Cette faible teneur en protéine de la farine de poisson serait due au choix du tissu du poisson utilisé ou à l'espèce de poisson car, la composition biochimique varie en fonction de ces critères sus-énumérés. Pour ce qui est du son de blé, la teneur en protéines $(14,97 \pm$ $0,16)$ est légèrement inférieure aux données courantes (Ngom, 2004). En effet, la teneur en protéines peut dépendre de l'efficacité du décorticage qui empêche que des grains entiers ou brisés se retrouvent dans le son, ce qui contribuerait à diminuer la teneur en protéine. De même, la teneur en protéine des maïs est similaire à la valeur $9,7 \%$ trouvée dans le maïs local du Sénégal (Mpouok, 1999). Parmi les huit acides aminés essentiels chez les animaux, la méthionine, la lysine et la thréonine font partie des acides aminés les plus pauvres dans les céréales (Fomekong et al., 2018) et les plus instables au 
cours des procédés de transformation. Les teneurs de ces trois acides aminés trouvés dans le soja, le tourteau de soja, le tourteau de coton et la farine de poisson, couvrent les besoins recommandés pour la nutrition des poulettes (INRA 2015). Les teneurs en matières grasses du tourteau de soja et de coton sont respectivement inférieures aux valeurs trouvées dans les différents types de tourteau de soja (Ponka et al., 2017) et de coton (Mongodin \& Rivière, 1965). Ces faibles teneurs en matières grasses du tourteau de soja et de coton peuvent s'expliquer par le fait que l'extraction d'huile des grains de soja et de coton a été optimale lors de la presse. La teneur en matières grasses du soja est légèrement supérieure à la valeur de $17 \%$ estimée dans la table de composition de la FAO (2019). Les teneurs en matières grasses des sons de riz et de blé sont respectivement supérieures aux valeurs trouvées dans les sons de riz et de blé au Sénégal (Mpouok, 1999). Cela pourrait être dû au fait que des germes se soient retrouvés dans les sons de riz et de blé lors du décorticage, ce qui contribuerait à augmenter la teneur en matières grasses. La teneur en matières grasses de la farine de poisson est comparable à celle trouvée $(10,9 \%)$ dans les farines de poisson en France (Bastianeli et al., 2009). Les teneurs en glucides $(73,8 \pm$ $0,07)$ et $(66,57 \pm 0,45)$ respectivement pour les maïs jaune et blanc sont proches de la valeur $(73,3 \%)$ trouvée dans le maïs de France (Bastianeli et al., 2009). mais reste inférieures à la valeur $(76,21)$ trouvée dans le maïs du Sénégal (Ngom, 2004). Ces teneurs sont relativement élevées, ce qui est appréciable étant donné que les glucides constituent la principale source d'énergie nécessaire au métabolisme de base et au fonctionnement du système nerveux (Ponka et al., 2017). Certains glucides participent à la constitution des cartilages, des acides nucléiques, du mucus, des glycoprotéines et des immunoglobulines des organismes (Sguera, 2008). II faut cependant noter qu'un aliment présentant un taux élevé en glucides n'est pas systématiquement un aliment ayant une forte valeur énergétique du fait qu'il y a des glucides digestibles et d'autres sont des fibres pour les monogastriques (Ponka et al., 2017). La teneur en cendres de la farine de poisson est supérieure à la valeur 18,98 $\pm 0,67 \%(\mathrm{~m} / \mathrm{m})$ trouvée dans les farines de poisson au Cameroun (Ponka et al., 2017), mais reste inférieure à la valeur (37\%) trouvée dans la farine de poisson de France (Bastianeli et al., 2009). Cette différence de teneur peut être liée à l'espèce de poisson utilisée pour fabriquer la farine de poisson. La teneur en cendres du tourteau de coton est comparable à celle $(7 \%)$ trouvée dans le tourteau de coton de la Cote d'Ivoire (Huart, 2004). La teneur en cendres du son de riz est inférieure à celle $(24,94 \%)$ trouvée dans le son de riz du Sénégal (Ngom, 2004). La farine de poisson est plus riche en cendres par rapport aux autres matières premières analysées. Cela traduit aussi une teneur élevée en sels minéraux qui sont des éléments indispensables au bon fonctionnement de l'organisme et à la croissance des volailles. La teneur en cellulose brute du tourteau de coton est supérieure à la valeur 12,95\% trouvée par Mongodin et Rivière (1965). La même observation est faite pour la valeur trouvée dans le son de blé $(8,9 \%, \mathrm{~m} / \mathrm{m})$ au Sénégal (Mpouok, 1999). Cette teneur élevée de la teneur de cellulose du son de blé peut être attribuée à la variété utilisée. Contrairement aux autres céréales, la teneur en cellulose du son de riz est comparable à la valeur trouvée dans le son de riz d'autres pays (Huart, 2004).

Le calcium est fortement concentré dans la coquille d'huitre et le phosphate bicalcique tandis que le phosphore est concentré dans le phosphate bicalcique. Ces chiffres sont similaires d'une part, aux teneurs de calcium de la coquille et du phosphate bicalcique et d'autre part à la teneur du phosphore du phosphate bicalcique rapportée par Huart (2004). Les valeurs énergétiques des maïs jaune et blanc, du son riz et de la farine de poisson sont comparables à celles trouvées par Huart (2004) pour les maïs jaune et blanc, le son de riz et la farine de poisson. Ce qui est intéressant est que la valeur énergétique de ces matières premières ci-dessus citées sont nettement supérieures à $280 \mathrm{kcal} / 100 \mathrm{~g}$ tel que recommandé par INRA (2015). Pour ce qui est des autres matières premières (tourteaux de soja et coton et le son de blé), la valeur énergétique est légèrement inférieure à la valeur recommandée par INRA (2015). En somme, la valeur énergétique élevée de certaines des matières premières analysées peut-être expliquée par le fait qu'elles ont une teneur élevée en lipides. Cependant, en alimentation animale, on dit d'un aliment qu'il est riche en énergie quand il renferme une teneur importante en glucide (surtout en amidon). Ainsi, les aliments énergétiques chez les monogastriques sont les céréales (maïs, sorgho, blé, riz, etc.). D'autre part, les régimes alimentaires pour les volailles sont composés principalement d'un mélange de plusieurs aliments tels que les céréales, tourteaux de soja, dérivés de produits animaux (farine de poisson, os, coquilles, etc.), de matières grasses, de pré-mélanges de vitamines et de minéraux. Tous ces aliments fournissent l'énergie et les nutriments nécessaires pour la croissance, la reproduction et le bien-être des animaux (Ponka et al., 2017). 


\section{CONCLUSION ET APPLICATION DES RESULTATS}

II ressort de cette étude que la farine de poisson présente les teneurs les plus élevées en protéine $(59,9 \pm$ $0,27 \mathrm{~g} / 100 \mathrm{~g})$, calcium $(6,42 \pm 0,04 \mathrm{~g} / 100 \mathrm{~g})$ et phosphore $(3,88 \pm 0,06 \mathrm{~g} / 100 \mathrm{~g})$. Le soja est le plus riche en matière grasse $(17,98 \pm 0,10 \mathrm{~g} / 100 \mathrm{~g})$ et le maïs jaune est le plus riche en glucide $(73,8 \pm 0,07 \mathrm{~g} / 100 \mathrm{~g})$. La caractérisation de ces matières premières a permis de mettre en évidence leur potentiel nutritionnel dans l'alimentation de la volaille au Burkina Faso. Ces données produites peuvent servir à l'établissement de tables de composition des matières premières locales utilisables dans l'alimentation de volaille et leur vulgarisation auprès des aviculteurs leur permettra de mieux s'orienter dans le choix des ingrédients de hautes valeurs nutritionnelles et de réduire le coût de la formulation.

\section{REMERCIEMENT}

La Société de Fabrique d'aliments pour Bétail SOFAB.SA est remerciée pour la collaboration dans le cadre des analyses. Le projet ISP/IPICS/RABIOTECH est remercié pour le soutien financier.

\section{BIBLIOGRAPHIE}

Afolayan MO and Afolayan M, 2008. Nigeria Oriented Poultry Feed Formulation Software Requirements. Journal of Applied Sciences Research, 4(11), 1596-1602. https://www.researchgate.net/publication/2286 52695

Almasad M, Altahat E, AL-Sharafat A, 2011. Applying Linear Programming Technique to Formulate Least Cost Balanced Ration for White Eggs Layers in Jordan. International Journal of Empirical Research, 1(1), 112-120.

AOAC, 1999. Official Methods of Analysis Chemists. Washington D.C. 808-1113

Aubry IM, 2012. Détermination de la teneur en cellulose brute dans les aliments pour animaux version date d' application. In Agence fédérale pour la Sécurité de la Chaîne alimentaire (Vol. 7, pp. 18).

Ayssiwede SB, Dieng A, Houinato MRB, Chrysostome CAAM, Issay Hornick JL, Missohou A, 2013. Elevage des poulets traditionels ou indigènes au Sénégal et en Afrique Subsahariene: État des lieux et contraintes. Annales de Medecine Veterinaire, 157(2), 103-119.

Bastianeli D, Omaria RE, Bonnal L, Grinaud P, 2009. Qualité des matières premières destinées a I' alimentation des volailles: Résultats d'une étude en Afrique de l'Est. Perspectives pour la gestion de la variabilité des matières premières. Revue Africaine de Santé et de Productions Animales, 7, 33-40.

Bindlingmeyer BA, Cohen SA, Tarvin TL, 1984. Rapid analysis of amino acids using precolumn derivitization. In J. Chomatogr.336
FAO (Organisation des Nations Unies pour l'Alimentation et l'Agriculture), 2018. Rapport Pays 20182050

FAO (Organisation des Nations Unies pour l'Alimentation et l'Agriculture), 2019. Table de composition des aliments FAO/INFOODS pour l'Afrique de l'Ouest (2019).

Fomekong TAM, Kana JR, Ngouana T.R, Mube K H, Teguia A. 2018. Effets du taux de la lysine et de la méthionine de la ration sur les performances de croissance de la poule Barrée du Cameroun. International Journal of Biological and Chemical $\begin{array}{lll}\text { Sciences, } & 12(2), & 927 .\end{array}$ https://doi.org/10.4314/ijbcs.v12i2.24

Houba VJ, Walinga I, Van der Lee JJ, Van Vark W, 1980. Plant analysis procedure (part 7, chapter 2. 3). Wageningen, The Netherlands. Department of Soil Sciences and plant Analysis.

Huart A, 2004. Les ingrédients qui composent I 'aliment volaille. Eco Congo, 1-5.

INRA,. 2015. Alimentation des volailles en agriculture biologique. Alimentation Des Volailles En Agriculture Biologique, 68. http://www.cfppaariegecomminges.fr/mogeli/A uto/docauto/Alimentation-Volailles-Bio.pdf

Mongodin B and Rivière R, 1965. Valeurs bromatologiques de 160 aliments de I' Ouest Africain. In Rev. El. Méd. Vét. 2, Issues 183218.

Montreuil J and Spik G, 1969. Micro dosage des glucides, méthodes colorimétriques de dosage des glucides totaux. France. 85 .

Mpouok O, 1999. Contribution a la mise au point d'un référentiel sur la qualité des matières premières utilisées en aviculture au Sénégal. Application 
a la formulation des rations alimentaires.

Ngom S, 2004. Ebauche d'un référentiel sur la composition chimique et valeur nutritive des matières premières utilisables en alimentation des volailles au Sénégal. 158.

Oladokun V and Johnson A, 2012. Feed formulation problem in Nigerian poultry farms: a mathematical programming approach. American Journal of Scientific and Industrial Research, 3(1), 14-20. https://doi.org/10.5251/ajsir.2012.3.1.14.20

Pomar C, Dibeau F, MILGEN V, 2009. La détermination des besoins nutritionnels, la formulation multicritère et l'ajustement progressif des apports de nutriments aux besoins des porcs: Des outils pour maitriser les rejets d'azote et de phosphore. INERA Productions Animales, 22(1), 49-54.

Ponka R, Goudoum A, Chami Tchungouelieu A, Fokou $E, 2017$. Evaluation nutritionnelle de quelques ingrédients entrant dans la formulation alimentaire des poules pondeuses et porcs d'une ferme d'élevage au Nord- Ouest Cameroun. International Journal of Biological and Chemical Sciences, 10(5), 2073. https://doi.org/10.4314/ijbcs.v10i5.11

Saxena P, 2012. Comparison of Linear and Nonlinear Programming Techniques for Animal Diet. Journal Applied Mathematics, 1(2), 106-108. https://doi.org/10.5923/j.am.20110102.17

Sguera S, 2008. Spirulina platensis et ses constituants : intérêts nutritionnels et activités thérapeutiques. In Sciences pharmaceutiques. https://hal.univ-lorraine.fr/hal-01732214 